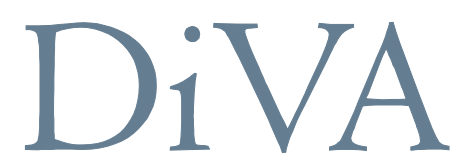

http://uu.diva-portal.org

This is an author-produced version of a paper published in IEEE Journal of Oceanic Engineering. This paper has been peer-reviewed but does not include the final publisher proof-corrections or journal pagination.

Citation for the published paper:

Yuen K., Thomas K., Grabbe M., Deglaire P., Bouquerel M., Österberg D., Leijon M.

"Matching a permanent magnet synchronous generator to a fixed pitch vertical axis turbine for marine current energy conversion"

IEEE Journal of Oceanic Engineering, 2009, 34(1), pp. 24-31

URL: http://dx.doi.org/10.1109/JOE.2008.2010658

(C) 2009 IEEE. Personal use of this material is permitted. Permission from IEEE must be obtained for all other uses, in any current or future media, including reprinting/republishing this material for advertising or promotional purposes, creating new collective works, for resale or redistribution to servers or lists, or reuse of any copyrighted component of this work in other works.

Access to the published version may require subscription. 


\title{
Matching a permanent magnet synchronous generator to a fixed pitch vertical axis turbine for marine current energy conversion
}

\author{
Katarina Yuen, Karin Thomas, Mårten Grabbe, Paul Deglaire, Mathias Bouquerel, David Österberg \\ and Mats Leijon, Member, IEEE
}

\begin{abstract}
Extracting energy from a free-flow marine current using a vertical axis fixed pitch turbine requires a generator that can handle varying speeds and loads, since such a turbine gives maximum power capture for a fixed tip speed ratio. A prototype of such a generator has been designed and constructed. In this paper, its variable speed and load operation is evaluated, both in terms of how the constructed generator performs in relation to simulations, and in terms of how the generator could perform with three different fixed pitch turbines. Measurements of RMS (root-mean-square) voltage and current differ $10 \%$ from simulations. Performance analysis with example turbines shows that the generator can match fixed tip speed ratio operation of several turbines for current velocities between 0.5 and $2.5 \mathrm{~m} / \mathrm{s}$.
\end{abstract}

\section{INTRODUCTION}

$\mathbf{F}$ REE flow marine currents, i.e. unregulated water courses, tides and other ocean currents, are an energy resource yet to be utilized on a commercial scale. Without dams, this nonfossil source can give an environmentally benign contribution to the world electricity production. The European tidal potential alone has been estimated to be 39-58 TWh annually [1]-[3]. A number of projects involved with extracting energy from this resource exist, and most concepts are similar to wind energy converters in that they include a turbine and a generator, e.g. [4]-[7].

Wind power is similar to marine current energy as it is concerned with harnessing the kinetic energy in a flowing fluid, and there are many relevant comparisons concerning underlying physics, practical engineering experience, etc. However, there are also a number of significant differences, e.g. resource characteristics. As most parts of the system will be submerged in water and thus not easily accessible, it is good to try to minimize needs for maintenance and repair by avoiding mechanically complex systems with many moving parts. In [8], it is concluded that up to 20 percent of the downtime for a wind power plant is due to gearbox failures, and that the majority of these failures are due to wear. Therefore, avoiding gearboxes, yawing and blade pitching is likely to be beneficial

Manuscript received December 14, 2007

Corresponding author is K. Yuen (e-mail: katarina.yuen@angstrom.uu.se, phone: +46184713368). All authors are with the Centre for Renewable Electric Energy Conversion at the Division of Electricity, Uppsala University, Box 534, 75121 Uppsala, Sweden.

The project is funded by Vattenfall AB, Uppsala University, The Swedish Agency for Innovation Systems (VINNOVA), The Swedish Energy Agency (STEM), Ångpanneföreningen's Foundation for Research and Development, The J. Gust Richert Foundation for Technical Scientific Research and CF's environmental fund.

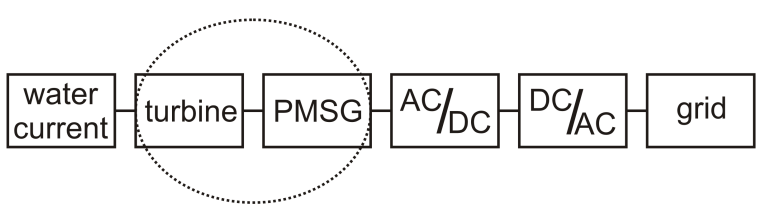

Fig. 1. A system for marine current energy conversion. The focus of this article is the connection between the turbine and the generator.

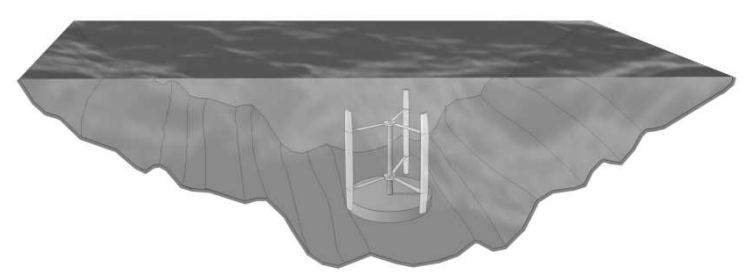

Fig. 2. Artist's impression of a vertical axis marine current energy converter.

for system reliability, but will require certain adaptations of the rest of the system. An example of such a system is illustrated in Fig. 1 and Fig. 2.

The purpose of this paper is to illustrate some of the consequences of variations in current velocities inherent to the resource, and show how a variable speed, direct drive permanent magnet synchronous generator (PMSG) specifically designed for marine current energy extraction with a fixed pitch vertical axis turbine can handle these variations.

\section{A. Resource characteristics}

For a given cross section, A, the power in a flowing fluid can be expressed as

$$
P=\frac{1}{2} \rho A v^{3}
$$

where $\rho$ is the density of the fluid and $v$ is its velocity [9]. The density of water is about $1000 \mathrm{~kg} / \mathrm{m}^{3}$, almost 800 times higher than that of air. A consequence of this is that water currents may be relevant for energy extraction even at low speeds, possibly down to the order of $1 \mathrm{~m} / \mathrm{s}$ or less, whereas in wind power a typical cut-in speed is in the range of $4 \mathrm{~m} / \mathrm{s}$ [10].

Equation (1) also shows that variations in energy flux will depend on the cube of the velocity. Variations in velocity 
of a marine current will typically be very predictable and depend mainly on the driving forces of the current and local bathymetry. Tidal currents will vary between standstill and maximum spring velocities that in a few extreme cases are up to $7 \mathrm{~m} / \mathrm{s}$, but in more locations are in the order of $2-3 \mathrm{~m} / \mathrm{s}$ [11]. For tides the direction of the flow will also vary. Velocities in rivers will depend on precipitation, season and local depth profiles. A system for extracting energy from free flow water currents should be able to handle the variations in power that these velocities correspond to.

\section{B. Variable speed operation with fixed pitch vertical axis turbine}

Efficient use of a turbine with fixed blade pitch in a varying flow speed requires variable speed operation. The power capture coefficient, $C_{p}$, for the turbine is usually expressed as a function of the tip speed ratio, $\lambda$

$$
\lambda=\frac{\Omega r}{v} .
$$

$\Omega$ is the rotational speed of the turbine and $r$ is the radius. Maximum power capture will typically be obtained at a specific tip speed ratio, $\lambda_{o p t}$, so keeping a fixed tip speed ratio will give maximum power capture. This means that the turbine should be operated at higher $\Omega$ for higher $v$, such that

$$
\Omega=\frac{v \lambda_{o p t}}{r},
$$

which will give power capture

$$
P_{t}=\frac{1}{2} \rho A v^{3} C_{p}^{\max }
$$

For a directly driven synchronous generator, the rotational speed of the generator will also be $\Omega$, and the speed of the turbine will be controlled through the generator. According to (4), the power absorbed by the turbine, and thus the input to the generator, is proportional to $v^{3}$, or $\Omega^{3}$.

Neglecting losses and assuming a resistive AC load, $R$, one can get a rough idea of how the generator needs to perform to match fixed tip speed ratio operation. The power output of the generator, which under the no-loss assumption should be equal to $P_{t}$, should be proportional to $\Omega^{3}$

$$
P_{t}=P_{g} \propto \Omega^{3}
$$

The power of the generator can be expressed as

$$
P_{g} \propto U I=\frac{U^{2}}{R} .
$$

Since the voltage $U$ is roughly proportional to $\Omega$ in a generator, one can see that the load should be allowed to vary as

$$
R \propto \frac{1}{\Omega}
$$

or that the current $I$ should vary such that

$$
I \propto \Omega^{2} .
$$

In practice, a variable speed generator that is to deliver power to the grid will be connected to a rectifier and inverter in order to adjust the voltage and frequency. In this case, the generator and turbine will be controlled through the inverter.

Taking losses into account, fixed tip speed ratio operation requires control of the generator so that

$$
P_{t}=P_{g}+P_{\text {loss }} \propto \Omega^{3} .
$$

Losses include mechanical, copper and iron losses. Copper losses in a permanent magnet machine are due to the resistance in the stator winding. Iron losses are mainly due to the hysteresis in magnetizing and demagnetizing soft magnetic materials in the machine and eddy currents induced in the stator steel. Copper, hysteresis and eddy current losses can be expressed as follows [12].

$$
\begin{gathered}
P_{C u}=R_{g} I^{2} \\
P_{\text {hysteresis }}=k_{h} B_{\text {max }}^{2} f \\
P_{\text {eddy }}=k_{e}\left(B_{\max } f\right)^{2}
\end{gathered}
$$

In the above equations, $R_{g}$ is the resistance in the armature winding, $k_{h}$ and $k_{e}$ are material constants, $B_{\max }$ is the maximum value of the magnetic flux density and $f$ is the electrical frequency. Since losses vary with rotational speed and load, (9) will not give a simple control scheme for the generator that gives fixed tip speed ratio operation.

Without knowledge of losses, fixed tip speed ratio operation can still be realized by continuous measurement of the current velocity and control of the rotational speed accordingly. Other control schemes for permanent magnet synchronous generators that do not require knowledge of the current velocity, such as maximum power point tracking (MPPT), vector control and control based on torque coefficient characteristics are discussed by e.g. [13]-[15] for wind energy converters. The object of this paper is however not to cover details in system control, but to relate generator performance to ideal turbine operation.

In water, a typical $\lambda_{o p t}$ can be around 2-3 for a fixed pitch vertical axis turbine [16]. A horizontal axis turbine with high solidity can also have a tip speed ratio as low as 1.2 [17]. Given that typical current velocities are low, i.e. around 2$3 \mathrm{~m} / \mathrm{s}$, one can see that the rotational speed of the turbine, and thus a direct drive generator, also will be low, according to (3). It is challenging to design electrical machines with high efficiency at such low speeds.

For a vertical axis turbine it is important to take the wake situation into account. One issue concerns the structural mechanics of the turbine. Another issue concerns farm implementation, where the wake from one turbine will affect other turbines. In both cases it is important to take into account the various unsteady effects due to the blades' interaction. As an illustration, detailed wake simulations for a turbine with 8 NACA0021 blades and a central tower have been carried out for the optimal tip speed ratio of 2.5. Fig. 3 shows detail of the wake close to the turbine. Fig. 4 shows the general wake characterization that needs to be taken into account in a farm implementation. The turbine used in these figures is example turbine $\mathrm{B}$, presented in section IV-B and Table II. In both figures red points represent clockwise vortex 


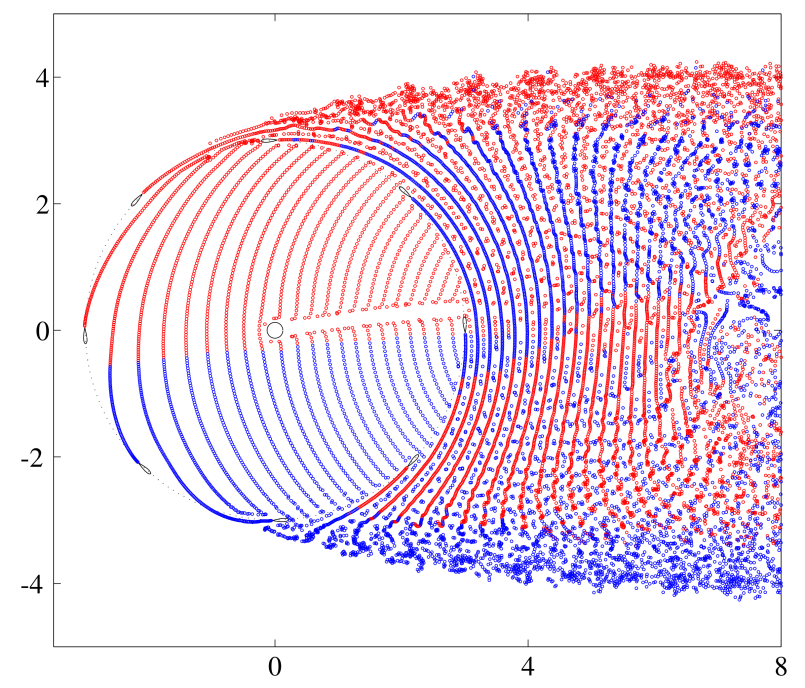

Fig. 3. Detailed wake simulation for a turbine with 8 NACA0021 blades and a central tower. Red points represent clockwise vortex particles and blue points counter-clockwise particles emitted from the blades. The unit on the axes is meters.

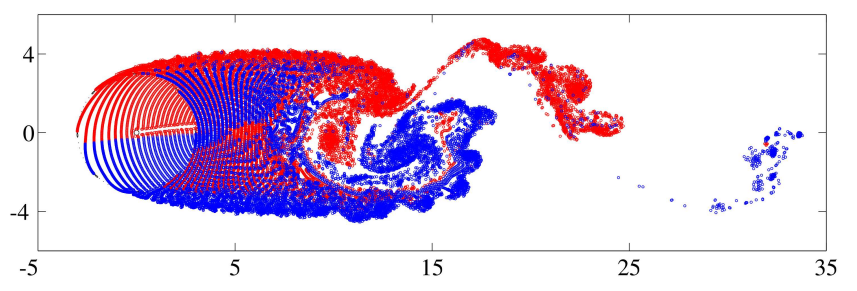

Fig. 4. General wake simulation for a turbine with 8 NACA0021 blades and a central tower. Red points represent clockwise vortex particles and blue points counter-clockwise particles emitted from the blades. The unit on the axes is meters.

particles and blue points counter-clockwise particles emitted from the blades. These simulations have been achieved using a two-dimensional model with innovative algorithms [18] and a new approach toward geometry using conformal mapping of multiply connected domains [19] resulting in a significant decrease in computation time.

\section{Concept studied at Uppsala University}

A system for extracting energy from marine currents is studied at Uppsala University, see Fig. 1 and 2. The turbine has a vertical axis and fixed blade pitch and therefore no yawing or blade pitch mechanisms. No gearbox is used as the turbine is connected directly to a permanent magnet synchronous generator, which in turn is connected to a rectifier and inverter, enabling control of the generator and turbine, as well as grid connection. The system as a whole is designed to have few moving parts in order to be robust and low-maintenance.

\section{DESIGN AND CONSTRUCTION OF GENERATOR FOR EXPERIMENTAL SETUP}

In [20], the design and construction of a prototype generator for laboratory testing is described. For a well known site and
TABLE I

GENERATOR DATA

\begin{tabular}{cc}
\hline \hline Design power & $5 \mathrm{~kW}$ \\
Design speed & $10 \mathrm{rpm}$ \\
Outer diameter & $2000 \mathrm{~mm}$ \\
Stator height & $270 \mathrm{~mm}$ \\
Air gap & $10.5 \mathrm{~mm}$ \\
Number of poles & 120 \\
Phases & 3 \\
Resistance in winding & $0.47 \Omega$ per phase \\
\hline \hline
\end{tabular}

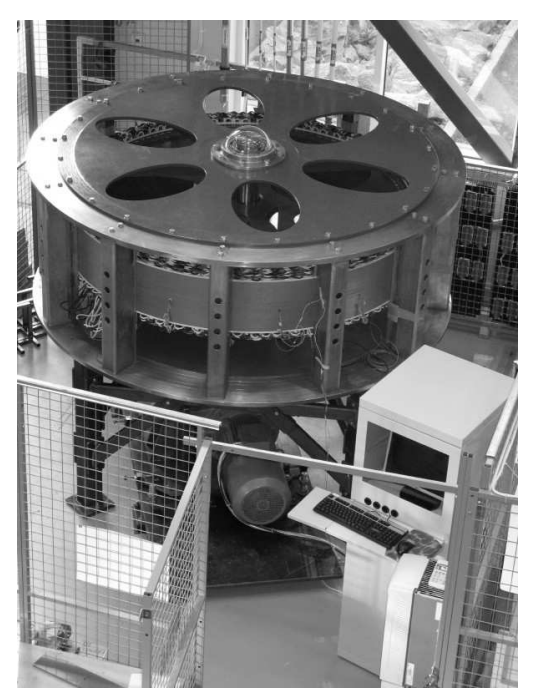

Fig. 5. Photograph of the experimental setup with prototype generator, artificial drive system and resistive load.

turbine design, it is possible to use the reasoning above to construct a generator for a specific range of speeds and loads. In this case, a turbine according to turbine $\mathrm{A}$ in table II, and a typical flow of $1.5 \mathrm{~m} / \mathrm{s}$ have been used to design the generator. For simplicity, initial simulations and testing are performed with a purely resistive AC load as suggested by [21].

\section{A. Electromagnetic design}

A finite element method (FEM) software tool originally made for $50 \mathrm{~Hz}$ machines has been used for the design and simulation of the generator. It calculates the magnetic and electric properties in a $2 \mathrm{D}$ generator geometry. A few design choices are $\mathrm{Nd}_{2} \mathrm{Fe}_{14} \mathrm{~B}$ magnets, $1 \mathrm{~mm}$ thick stator laminations and cable windings, and the generator has no cooling system. See Table I and [22] for more details. In this design process, the use of 'rated', or design power and speed differs somewhat from conventional use of the terms. Here, they refer to typical operational values rather than upper limits.

The upper limits of the range of operation for the generator will most likely be set by thermal and electrical properties of the winding insulation. The generator is wound with a standard cable, MK 450/750 [23], which is specified to handle RMS (root-mean-square) voltages of $450 \mathrm{~V}$ and temperatures of $70^{\circ} \mathrm{C}$ during continuous operation. 


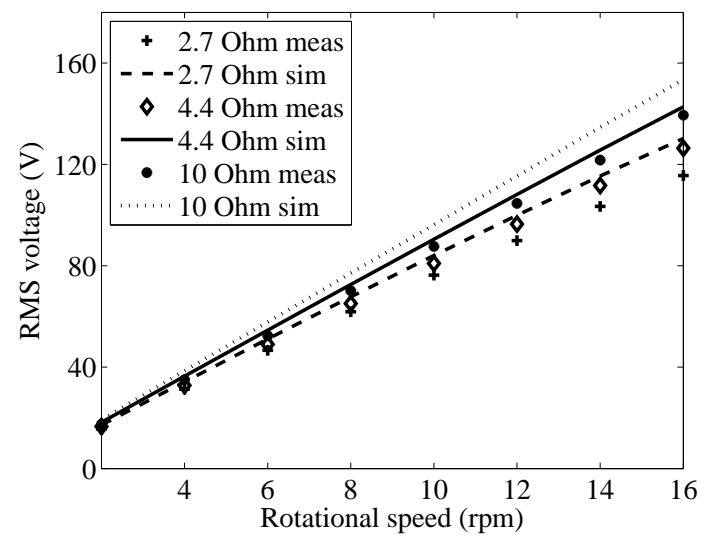

Fig. 6. Measured and simulated values of $U_{r m s}$ for various loads and speeds.

\section{B. Experimental setup}

The prototype generator is part of an experimental setup, see Fig. 5, with an artificial drive system including an induction motor and gearbox that allow operation from 0 to $16 \mathrm{rpm}$, a maximum torque of $11 \mathrm{kN} \cdot \mathrm{m}$ and power input up to $22 \mathrm{~kW}$. The power from the generator is spent in a resistive load that consists of a number of resistors that are connected in various configurations to give different load cases. The load can be varied between 10 and $2.7 \Omega$ per phase. The experimental setup is described further in [22].

\section{Measurements}

A metrix multimeter clamp MX240/MX2040 (accuracy \pm $(1 \% \mathrm{R}+8 \mathrm{D}))$ and a Le Croy Wave Surfer $424200 \mathrm{MHz}$ Oscilloscope (accuracy $\pm(1.5 \%+0.5 \%$ of full scale $))$ were used for measuring current and voltage. Previous measurements [20] have shown that the three phases of the constructed generator are well balanced. Therefore, measurements of voltage and current were limited to one phase. Voltage and current were measured for 2-16 rpm for four different load cases: 2.7, 4.4, 8 and $10 \Omega$ per phase. The specific load values are determined by possible configurations of the resistors included in the experimental setup, and are chosen to illustrate a range of loads higher and lower than the design load of $4.4 \Omega$. Simulations of the same load and speed cases were performed.

The experimental setup has yet to be equipped with torque measurements, so the total losses were not measured at this time. Resistive losses in the armature winding can be calculated based on the current and the measured resistance of the winding. Before magnetizing the rotor, frictional losses were calculated by measuring the deceleration of the rotor in accordance with IEEE Std 115-1995. The friction, including windage, was found to be approximately $2 \mathrm{~N} \cdot \mathrm{m}$ in the range of 0 to $20 \mathrm{rpm}$. Although the friction is expected to have increased somewhat after magnetization due to radial forces on the bearings, frictional losses are still expected to be small compared to the power output and are thus neglected in the following sections.

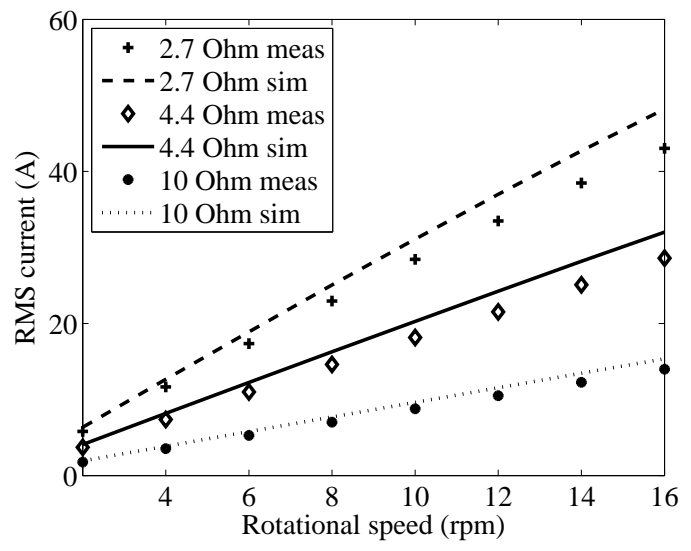

Fig. 7. Measured and simulated values of $I_{r m s}$ for various loads and speeds.

TABLE II

EXAMPLE TURBINES

\begin{tabular}{cccc}
\hline \hline Turbine & $\mathrm{A}$ & $\mathrm{B}$ & $\mathrm{C}$ \\
\hline Radius $(\mathrm{m})$ & 2.5 & 3 & 3 \\
Height $(\mathrm{m})$ & 1.6 & 3 & 3 \\
$\lambda_{\text {opt }}$ & 1.7 & 2.5 & 3 \\
$C_{p}\left(\right.$ at $\left.\lambda_{\text {opt }}\right)$ & 0.35 & 0.35 & 0.35 \\
\hline \hline
\end{tabular}

\section{RESUlTS AND DisCUSSION}

\section{A. Comparison of measured and simulated data}

The RMS voltage $\left(U_{r m s}\right)$ and current $\left(I_{r m s}\right)$ of the generator as a function of the rotational speed are shown for three load cases in Fig. 6 and Fig. 7. Measured values of both $U_{r m s}$ and $I_{r m s}$ are $10 \%$ lower than simulated values. Measured $U_{r m s}$ vary from 84 to $92 \%$ of simulated values (standard deviation $1.3 \%$ ) and measured $I_{r m s}$ vary from 85 to $92 \%$ of simulated values (standard deviation 1.3\%). Peak to peak voltages show better agreement, i.e. measured values of $U_{p p}$ are on average $6 \%$ lower than simulations. The simulations show a higher content of harmonics than measurements, which explains the difference in agreement between RMS and peak to peak values. Differences between the measurements and simulations are attributed to measurement uncertainties and inaccuracies in modeling the actual generator.

\section{B. Generator performance with example turbines}

In order to relate the performance of the generator to a setting with a turbine in a water current, three example vertical axis turbines with fixed blade pitch are used, see Table II. Turbine A, which is the fictive turbine used in dimensioning the generator, has a high solidity, i.e. a long blade chord length in relation to the turbine radius, as in [17]. Turbine B and $\mathrm{C}$ have higher optimal tip speed ratios and a larger cross section than turbine A. Turbine B is designed with 8 blades with chord length of $0.25 \mathrm{~m}$, while turbine $\mathrm{C}$ has 6 blades with chord length $0.23 \mathrm{~m}$. The $C_{p}$-curves in Fig. 4 have been generated by a double multiple stream tube model including secondary effects (flow curvature, dynamic stall, edge effects, strut losses) and based on a local flow description [24]. This 


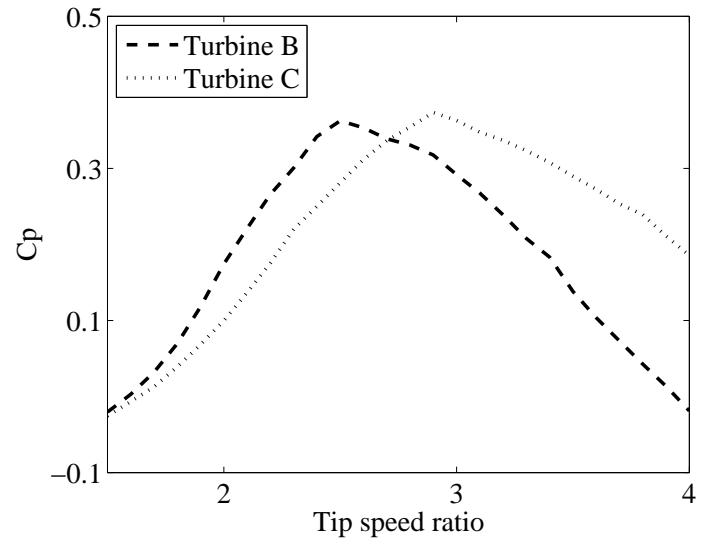

Fig. 8. $C_{p}$-curves for two example turbines.

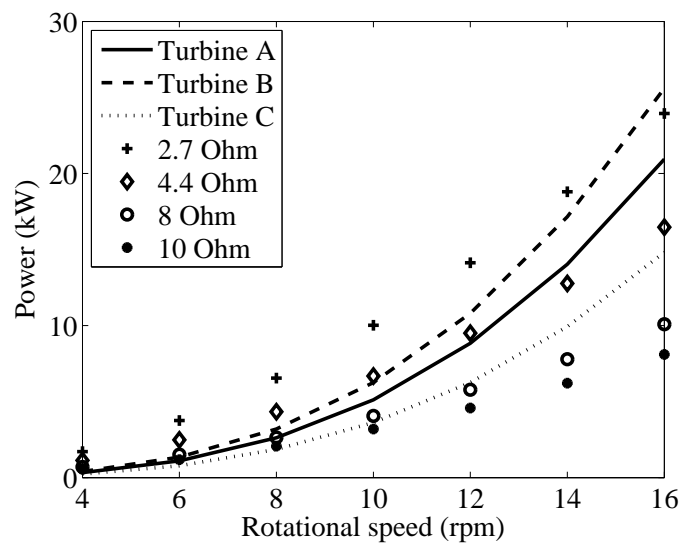

Fig. 9. Power output of three turbines and electromagnetic power of the generator (power output of generator plus copper and iron losses) for different loads.

tool has been validated in the case of vertical axis wind turbines as a good power curve estimation tool. These results have been compared to another three dimensional stream tube model [25] with very good agreement. Turbine B has been used in the generation of Fig. 3 and 4.

In Fig. 9 simulated values of the electromagnetic power for four different loads, including power output, copper losses and iron losses, are shown together with the power capture of the three example turbines when run at constant tip speed ratio, $\lambda_{\text {opt }}$. It is clear that the generator can accommodate the turbine it was designed for, but also other turbines. For turbines A, $\mathrm{B}$ and $\mathrm{C}$, operation at $16 \mathrm{rpm}$ corresponds to current speeds of $2.5,2$ and $1.7 \mathrm{~m} / \mathrm{s}$ respectively. Fig. 9 shows that a single generator with a wide range of operation in terms of speed and power can be used with various turbines, in various water currents.

In Fig. 10 the power capture of turbine A is shown together with simulated generator performance. To the left data is presented with a log scale to highlight the loss characteristics at low speeds. A suitable load has been chosen for each rotational speed corresponding to fixed tip speed ratio operation. Fig. 11 shows the electromagnetic efficiency and the RMS current of the generator corresponding to Fig. 10. Figures 12 and 13 show
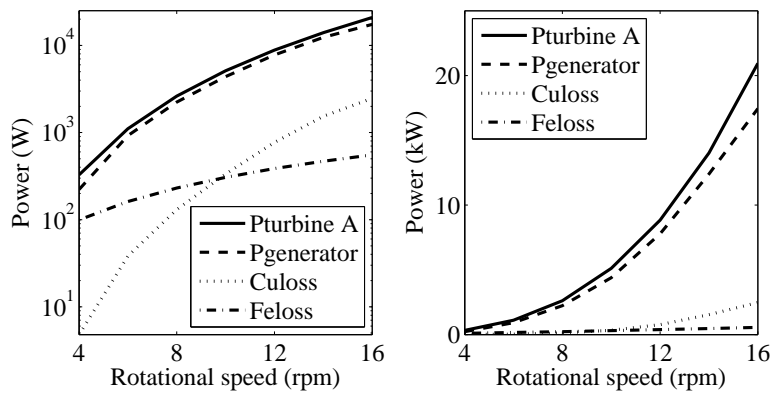

Fig. 10. Generator performance when matching fixed tip speed ratio operation of turbine A. For each speed, the load has been chosen so that the sum of output power and losses for the generator (based on simulations) equal the turbine power. Rotational speeds of 4-16 rpm correspond to water current speeds of $0.6-2.5 \mathrm{~m} / \mathrm{s}$. The graphs show the same data, but to the left in logarithmic scale and to the right linear. Iron losses exceed copper losses at low speeds, as can be seen in the left-hand graph.
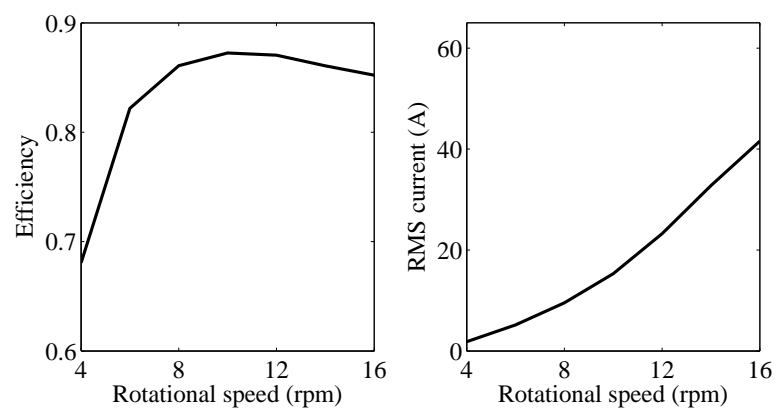

Fig. 11. Efficiency and RMS current of generator corresponding to Fig. 10 , i.e. when operated to match fixed tip speed ratio operation of turbine A. Rotational speeds of 4-16 rpm correspond to water current speeds of 0.6$2.5 \mathrm{~m} / \mathrm{s}$.

the same data when operating the generator to give fixed tip speed ratio operation of turbine $\mathrm{B}$.

Iron losses are the main cause of the reduced efficiency for low rotational speeds seen in Fig. 11 and 13. A rotational speed of $2 \mathrm{rpm}$ corresponds to $0.31 \mathrm{~m} / \mathrm{s}$ for turbine A and $0.25 \mathrm{~m} / \mathrm{s}$ for turbine B. At that rotational speed, total iron losses are approximately equal to the power capture of the turbines, but at $4 \mathrm{rpm}$ an efficiency of about $70 \%$ can be obtained for the generator. At low speeds, the electrical frequency is also low, and the iron losses are mainly explained by the size of the stator. The fact that the iron losses are high relative to the power output is due to the low power flux in such a slow water current.

The electrical current in the winding increases approximately as $\Omega^{2}$, see Fig. 11 and 13, which means that the copper losses increase as $\Omega^{4}$ according to (10), and is the main reason for the lower efficiency at high rotational speeds in Fig. 13. Iron losses are frequency dependent, and the frequency is proportional to the rotational speed. Therefore, hysteresis losses increase with $\Omega$ and eddy current losses with $\Omega^{2}$. However, the power captured by the turbine increases with $\Omega^{3}$ and the iron losses do not dominate at higher speeds.

The combination of turbine and generator strongly affects the overall performance of the system, and the choice of generator and turbine design can be made to suit a specific site 

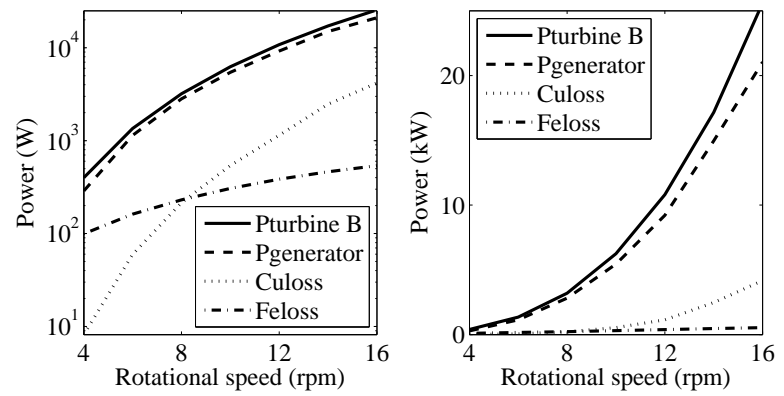

Fig. 12. Generator performance when matching fixed tip speed ratio operation of turbine B. For each speed, the load has been chosen so that the sum of output power and losses for the generator (based on simulations) equal the turbine power. Rotational speeds of 4-16 rpm correspond to water current speeds of $0.5-2.0 \mathrm{~m} / \mathrm{s}$. The graphs show the same data, but to the left in logarithmic scale and to the right linear. Iron losses exceed copper losses at low speeds, as can be seen in the left-hand graph.
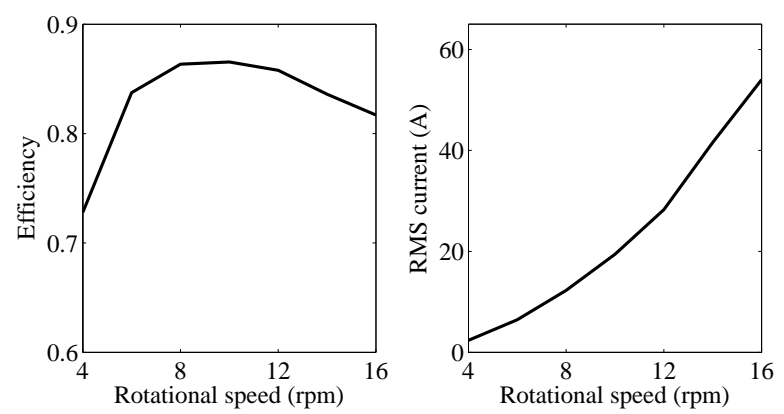

Fig. 13. Efficiency and RMS current of generator corresponding to Fig. 12 , i.e. when operated to match fixed tip speed ratio operation of turbine $B$. Rotational speeds of 4-16 rpm correspond to water current speeds of $0.5-$ $2.0 \mathrm{~m} / \mathrm{s}$.

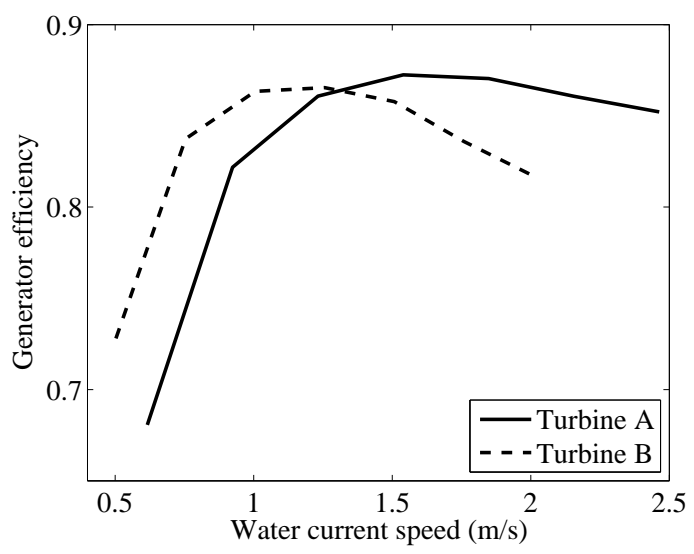

Fig. 14. Generator efficiency versus water current speed when the generator is run according to optimal tip speed ratio with turbine $\mathrm{A}$ and $\mathrm{B}$.

with known bathymetry and water current velocity variations. As can be seen in Fig. 14, operation with two different turbines results in different generator efficiencies. The combination with the prototype generator and turbine $\mathrm{B}$ gives good performance at slower water current speeds than the combination with turbine A. Apart from the fact that the turbines have different cross sections, turbine B would be a better choice at a site where water currents often are below $1 \mathrm{~m} / \mathrm{s}$ but seldom

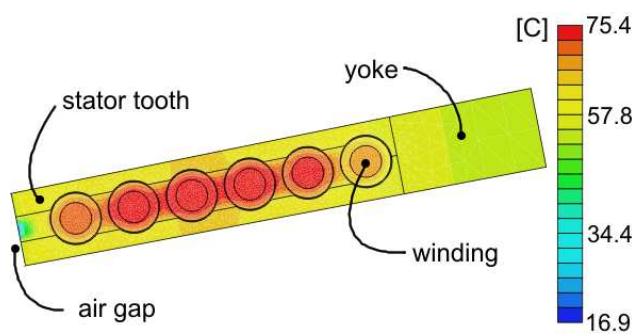

Fig. 15. Temperature in a segment of the stator. The figure shows a single stator slot and halves of the adjoining teeth. The simulation is for $10 \mathrm{rpm}$ with $0.001 \Omega$ per phase load, resulting in an RMS current of $I_{e} \approx 60 \mathrm{~A}$. Maximum temperatures of approximately $70^{\circ} \mathrm{C}$ are reached by the winding in the middle of the slot.

exceed $1.5 \mathrm{~m} / \mathrm{s}$. Operation with both turbines, though more pronounced with turbine A, results in a wide efficiency 'peak' as can be seen in Fig. 14, i.e. an efficiency of about $80 \%$ or more for a wide range of speeds. One can also note that in general, electrical machines with low power rating have lower efficiency.

\section{Upper limits of operation}

As mentioned in section II, the upper limits of the range of operation for the generator are expected to be given by the winding insulation. Fig. 6 shows that the voltage stays well within the range of what the insulation can handle during these circumstances, as expected, and fixed tip speed ratio operation implies that the load increases with the rotational speed, thus limiting the voltage.

The measurements presented in Fig. 6 and Fig. 7 did not pose any temperature problems for the generator. In order to find a rough upper limit concerning temperature, analysis was performed using the simulation tool. As the generator has no active cooling mechanism, cooling takes place through heat transfer to the surroundings and the temperature will be determined by heat transfer and losses. In general, losses increase with rotational speed and load. Under such circumstances, resistive losses dominate, as can be seen in Fig. 10 and 12, and therefore, the stator temperature is expected to be affected mainly by the electrical current in the armature winding. Simulations show that continuous operation with an electrical current of approximately $60 \mathrm{~A}$ and an ambient temperature of $20^{\circ} \mathrm{C}$ will lead to maximum temperatures in the stator close to $70^{\circ} \mathrm{C}$, see Fig. 15 . This simulation should ideally be calibrated with measurements of actual heat transfer, and will in an outdoor setting be affected by ambient water temperatures and the design of the generator housing.

Considering the turbine, the rotational speed should be limited in order to prevent cavitation of the turbine blades. Cavitation causes wear of the turbine through the collapse of small vapor pockets that are created when the speed of the blades is high enough to cause local pressure drops below the vapor pressure of the water. The exact limitation of rotational speed set by cavitation depends on a number of parameters including water depth and turbine radius, but as a rule of thumb, [1] suggests an upper limit of the relative velocity of the blades of $7 \mathrm{~m} / \mathrm{s}$. Structural mechanics issues may also limit the rotational speed of the turbine. 
For the system as a whole it is not possible to determine a general upper limit for rotational or water current speeds since the turbine characteristics affect how the generator should be operated. For a specific turbine, generator and site, these limitations can be determined and guide the control of the system. In practice, if water current speeds become too high, the turbine can be operated at a lower tip speed ratio than $\lambda_{o p t}$, in order to limit rotational speed and armature current.

For the example turbines presented here, one can see in Fig. 13 that fixed tip speed ratio operation of turbine $\mathrm{B}$ can result in RMS currents of about $54 \mathrm{~A}$ at $16 \mathrm{rpm}$. In an outdoor setting, the cooling may be improved by ambient water temperatures being lower than the $20^{\circ} \mathrm{C}$ used in the simulation, allowing higher electrical currents than $60 \mathrm{~A}$. The maximum relative velocity for turbine $B$ at $16 \mathrm{rpm}$ is approximately $7 \mathrm{~m} / \mathrm{s}$. In combining the prototype generator and turbine $\mathrm{B}, 16 \mathrm{rpm}$, or $2 \mathrm{~m} / \mathrm{s}$, is approximately where it is appropriate to stop operating the turbine at $\lambda_{\text {opt }}$.

Economical considerations have not been highlighted in this article, but will of course guide the choice of installed power capacity for a given site. Installing generators and turbines that give best efficiency at maximum speeds may not be the most economical choice as illustrated for tides in [26].

\section{CONCLUSION}

A generator suitable for variable speed and load operation has been designed and constructed. A comparison of measurements and simulations of effective values of voltage and current show that measurements are $90 \%$ of simulated values.

Comparisons with three example fixed pitch vertical axis turbines show that the prototype generator can match fixed tip speed ratio operation of several turbines for current speeds between approximately 0.5 and $2.5 \mathrm{~m} / \mathrm{s}$. For other water current speeds, a different combination of turbine and generator may be more suitable.

\section{ACKNOWLEDGMENTS}

Arne Wolfbrandt and Karl-Erik Karlsson are hereby acknowledged for development of the simulation tool. Sincere thanks to Ulf Ring for supervision and assistance in the construction work.

\section{REFERENCES}

[1] IT Power Ltd, "Non nuclear energy - JOULE II, wave energy project results, the exploitation of tidal marine currents," EU JOULE contract JOU2-CT94-0355, 1996.

[2] Black \& Veatch, "Tidal stream resource report," Peer Review Issue 1, Report Submission 107799/D/2100/05/1, 2004.

[3] Department of Trade and Industry, UK, "Tidal stream energy review," ETSU-T/05/00155/REP, 1993.

[4] E. 21616, "Seaflow - world's first pilot project for the exploitation of marine currents at a commercial scale," ISBN: 92-894-4593-9, pp. 1-34, 2005.

[5] "Clean Current," http://www.cleancurrent.com, Dec. 2007.

[6] "Blue Energy," http://www.bluenergy.com/technology.html, Dec. 2007.

[7] S. E. Ben Elghali, R. Balme, K. Le Saux, M. E. H. Benbouzid, J. F. Charpentier, and F. Hauville, "A simulation model for the evaluation of the electrical power potential harnessed by a marine current turbine," IEEE Journal of Oceanic Engineering, vol. 32, no. 4, pp. 786-789, Oct. 2007, 10.1109/JOE.2007.906381.
[8] J. Ribrant and L. Bertling, "Survey of failures in wind power systems with focus on Swedish wind power plants during 1997-2005," IEEE Transactions on Energy Conversion, vol. 22, no. 1, pp. 167-173, Mar. 2007.

[9] J. Manwell, J. McGowan, and A. Rogers, Wind Energy Explained Theory, Design and Application. West Sussex, England: John Wiley \& Sons Ltd., 2002, ch. 2.2.3.1, p. 31.

[10] Vestas, "Product brochure Vestas V80-2 MW," http://www.vestas.com/, Nov. 2007.

[11] I. G. Bryden and S. Couch, "ME1-marine energy extraction: tidal resouce analysis," Renewable Energy, vol. 31, no. 2, pp. 133-139, Feb. 2006.

[12] K.-E. Hallenius, Elektriska maskiner. Lund, Sweden: CWK Gleerup Bokförlag, 1972, ch. 3, pp. 3-24-29.

[13] I. Schiemenz and M. Stiebler, "Control of a permanent magnet synchronous generator used in a variable speed wind energy system," Proceedings of IEEE International Conference on Electric Machines and Drives, IEMDC 2001, pp. 872-877, 2001.

[14] G. Ramtharan, N. Jenkins, and O. Anaya-Lara, "Modelling and control of synchronous generators for wide-range variable-speed wind turbines," Wind Energy, vol. 10, no. 3, pp. 231-246, Mar. 2007.

[15] T. Wakui and R. Yokoyama, "A suitable load control method for constant tip speed ratio operation of stand-alone wind turbine-generator systems (Part I: Evaluation of power generation capability and output fluctuation)," Wind Engineering, vol. 31, no. 1, pp. 43-58, 2007.

[16] S. Antheaume, T. Maître, C. Buvat, and C. Abonnel, "Optimization of a vertical axis water turbine farm," International Conference - from Innovation to Industry, Bremerhaven, Germany, pp. 1-8, Oct. 2006.

[17] J. A. V. Alé, M. R. Petry, S. B. Garcia, G. C. da Silva Simioni, and G. Konzen, "Performance evaluation of the next generation of small vertical axis wind turbine," EWEC 2007 - European Wind Energy Conference \& Exhibition, pp. 1-10, May 2007.

[18] D. Österberg, "Multi-body unsteady aerodynamics in 2D using a vortex method," Master of Science Thesis, Uppsala University, Sweden, Dec. 2007.

[19] P. Deglaire, O. Ågren, H. Bernhoff, and M. Leijon, "Conformal mapping and efficient boundary element method without boundary elements for fast vortex particle simulations," European Journal of Mechanics B/Fluids, Apr. 2007

[20] K. Nilsson, K. Yuen, M. Grabbe, and M. Leijon, "A direct drive generator for marine current energy conversion - first experimental results," Proceedings of the 7th European Wave and Tidal Energy Conference, EWTEC 2007, pp. 1-5, Sep. 2007.

[21] I. Boldea, Variable Speed Generators. Boca Raton, Florida: CRC Press, Taylor \& Francis Group, 2006, sec. 10.15.6, p. 10.78.

[22] K. Thomas, M. Grabbe, K. Yuen, and M. Leijon, "A low speed generator for energy conversion from marine currents - experimental validation of simulations," Journal of Power and Energy (Part A of the Proceedings of the Institution of Mechanical Engineers), vol. 222, no. 4, pp. 381-388, 2008.

[23] Draka Kabel Sverige AB, "Data sheet for MK 450/750 cable," http://www.draka.se/Portals/0/Produkter/Pdf/MK_450750_V.pdf, Nov. 2007.

[24] A. Solum, P. Deglaire, S. Eriksson, M. Stålberg, M. Leijon, and H. Bernhoff, "Design of a $12 \mathrm{~kW}$ vertical axis wind turbine equipped with a direct driven PM synchronous generator," EWEC 2006 - European Wind Energy Conference \& Exhibition, Feb. 2006.

[25] G. F. Homicz, "VAWT (Vertical-Axis Wind Turbine) stochastic loads produced by atmospheric turbulence," 7th Wind Energy Symposium, Jan. 1988.

[26] J. A. Clarke, G. Connor, A. D. Grant, and C. M. Johnstone, "Regulating the output characteristics of tidal current power stations to facilitate better base load matching over the lunar cycle," Renewable Energy, vol. 31, no. 2, pp. 173-180, Feb. 2006. 


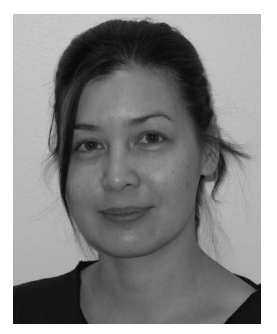

Katarina Yuen was born in her grandmother's house in 1976. She completed an M.Sc. in Engineering Physics at Uppsala University, Uppsala, Sweden in 2001. In 2006, after several years working with radiation protection, she joined the Division of Electricity at Uppsala University as a Ph.D. student within the marine current energy group.

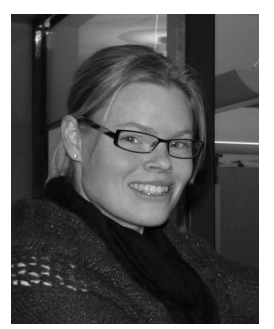

Karin Thomas (earlier Nilsson), received an M.Sc. in Engineering Physics at Uppsala University, Uppsala, Sweden, in 2003. In 2008 she received a Ph.D. in electrical engineering. Currently, she is working as a research associate at the Division of Electricity at Uppsala University.

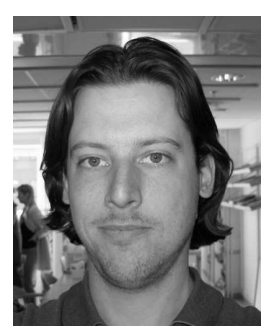

Mårten Grabbe, born in Sandviken, Sweden. He completed an M.Sc. degree in Engineering Physics at Uppsala University, Uppsala, Sweden in 2006. Currently he is working toward a licentiate degree at the Division of Electricity at Uppsala University.

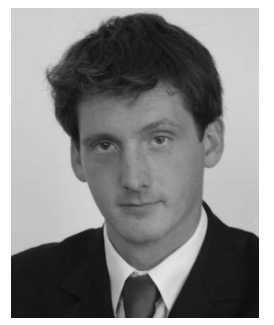

Paul Deglaire, born in Paris, France in 1978. Received M.Sc. degree in Engineering Physics from the École des Ponts et Chaussées, France in 2004. The author's major field of study is aerodynamics, vortex methods and fluid structure coupling applied to energy conversions for renewable energy applications. He has a position as a Ph.D. student at the Division of Electricity at Uppsala University and is involved in a vertical axis wind turbine project.

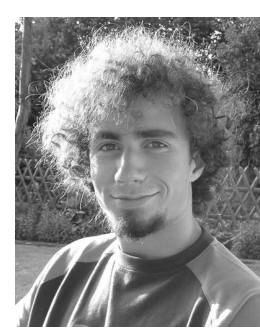

Mathias Bouquerel was born in Alenon, France, in 1985. Student at École des Ponts ParisTech (Paris, France), he is currently working toward an M.Sc. degree in Engineering Physics. During 2007-2008 he spent a year at the division of Electricity at Uppsala University, developing aerodynamics and structural mechanics tools for the wind power and current power projects.

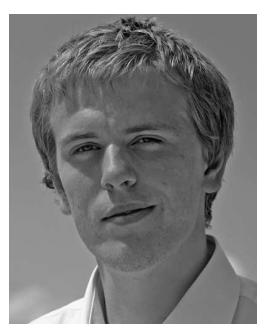

David Österberg conducted his M.Sc. thesis at the Division of Electricity at Uppsala University, Uppsala, Sweden in 2007. He is currently working at a company developing vertical axis wind turbines.

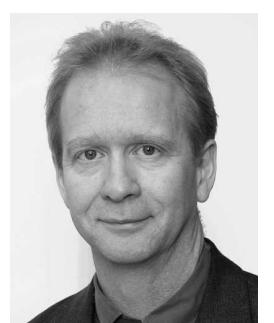

Mats Leijon (M83) received his Ph.D. in 1987 from Chalmers University of Technology, Gothenburg, Sweden.

From 1993 to 2000 he was head of the department for High Voltage Electromagnetic Systems at ABB Corporate Research, Västerås, Sweden. In 2000 he became professor of Electricity at Uppsala University, Uppsala, Sweden.

Dr. Leijon received the Chalmers award 'John Ericsson medal' in 1984, the 'Porjus International Hydro Power Prize' in 1998, the Royal University of Technology 'Grand Prize' in 1998, the Finnish academy of science 'Walter Alstrom prize' in 1999, the 2000 'Chalmers Gustav Dahlen medal', and the 'Polhem prize 2001' for the Powerformer technology. Dr. Leijon is a member of the Swedish Royal Academy of Engineering Science, WEC and Cigre. 